\title{
The RUTH Gripper: Systematic Object-Invariant Prehensile In-Hand Manipulation via Reconfigurable Underactuation
}

\author{
Quijie Lu, Nicholas Baron, Angus B. Clark, and Nicolas Rojas \\ REDS Lab, Dyson School of Design Engineering, Imperial College London \\ Email: \{q.lu17, n.baron17, a.clark17, n.rojas\}@imperial.ac.uk
}
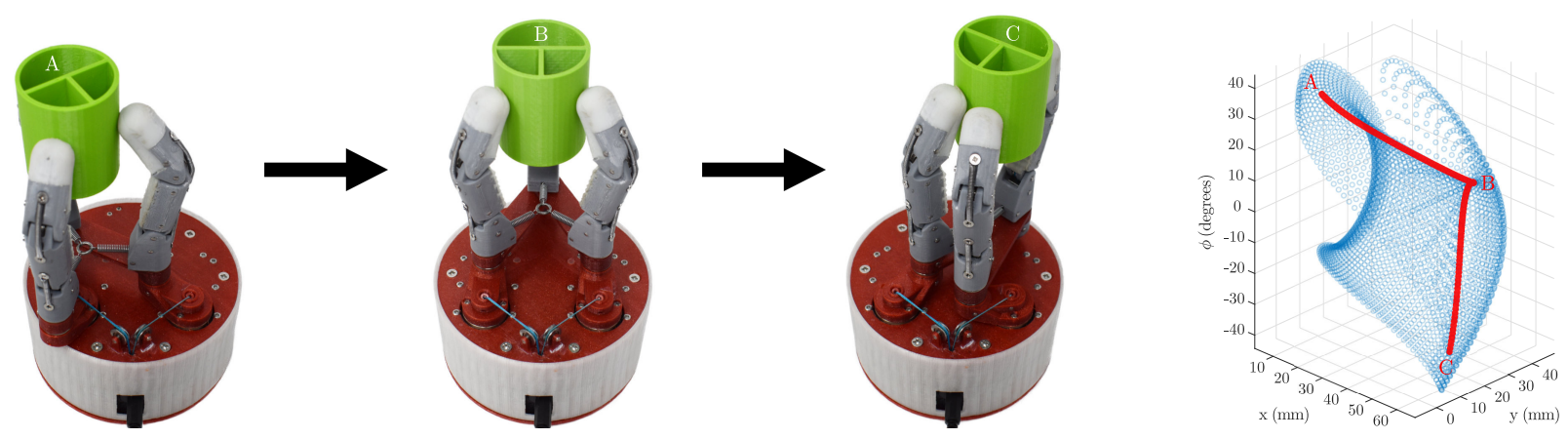

Fig. 1. The RUTH gripper decouples grasping and in-hand manipulation by exploiting palm reconfiguration and self-adaptiveness of underactuated fingers. With this gripper the motion of grasped objects of different sizes and shapes from one pose to another is straightforward and systematic, since an object-invariant map between task space and joint space can be easily pre-computed. The supplementary video can be found by this link https://imperialcollegelondon.box. com/s/1rl42qsv3y2cdchgb4ky4l3jiper41di Left: The RUTH gripper manipulating a cylindrical object from pose A to poses B and C. Right: The manipulation map with the numerical object trajectory from A to B to C.

Abstract-We introduce a reconfigurable underactuated robot hand able to perform systematic prehensile in-hand manipulations regardless of object size or shape. The hand utilises a two-degree-of-freedom five-bar linkage as the palm of the gripper, with three three-phalanx underactuated fingers-jointly controlled by a single actuator-connected to the mobile revolute joints of the palm. Three actuators are used in the robot hand system, one for controlling the force exerted on objects by the fingers and two for changing the configuration of the palm. This novel layout allows decoupling grasping and manipulation, facilitating the planning and execution of in-hand manipulation operations. The reconfigurable palm provides the hand with large grasping versatility, and allows easy computation of a map between task space and joint space for manipulation based on distance-based linkage kinematics. The motion of objects of different sizes and shapes from one pose to another is then straightforward and systematic, provided the objects are kept grasped. This is guaranteed independently and passively by the underactuated fingers using a custom tendon routing method, which allows no tendon length variation when the relative finger base position changes with palm reconfigurations. We analyse the theoretical grasping workspace and manipulation capability of the hand, present algorithms for computing the manipulation map and in-hand manipulation planning, and evaluate all these experimentally. Numerical and empirical results of several manipulation trajectories with objects of different size and shape clearly demonstrate the viability of the proposed concept.

\section{INTRODUCTION}

Prehensile in-hand manipulation involves manipulating a grasped object by a robot hand's fingers without losing contact with it. With the rising interest in robot hands, as an approach to achieve task versatility in robotic systems, not only robust grasping, but also in-hand manipulation has become an important and essential ability to improve dexterity. Several highly articulated anthropomorphic hands, with high number of degrees of freedom, have been indeed developed to achieve grasping and manipulation tasks [1, 2]. These robot hands are usually redundant by having actuators at each joint of the fingers, making them well suited to perform hand gestures but not necessarily reliable for prehensile in-hand manipulation as they become prone to error because of the large number of actuators. By introducing tendon driven and joint coupling design [3, 4], robot hands have been undergoing continuous improvements in performance and durability to mitigate these issues. Regarding state-of-the-art control strategies, deep reinforcement learning has been recently used to perform succesfully complex manipulation tasks with multifingered robot hands [5, 6], but the method has shown to require huge amounts of feedback data, and enormous time and energy consumption to achieve goals - with a relative low success rate and no fingertip force modulation. Indeed, performing reliable prehensile in-hand manipulation under both shape diversity and shape uncertainty with a robot hand is still an open problem [7].

Underactuated robot hands, by actuating multiple finger phalanges with a single actuator through a carefully designed 

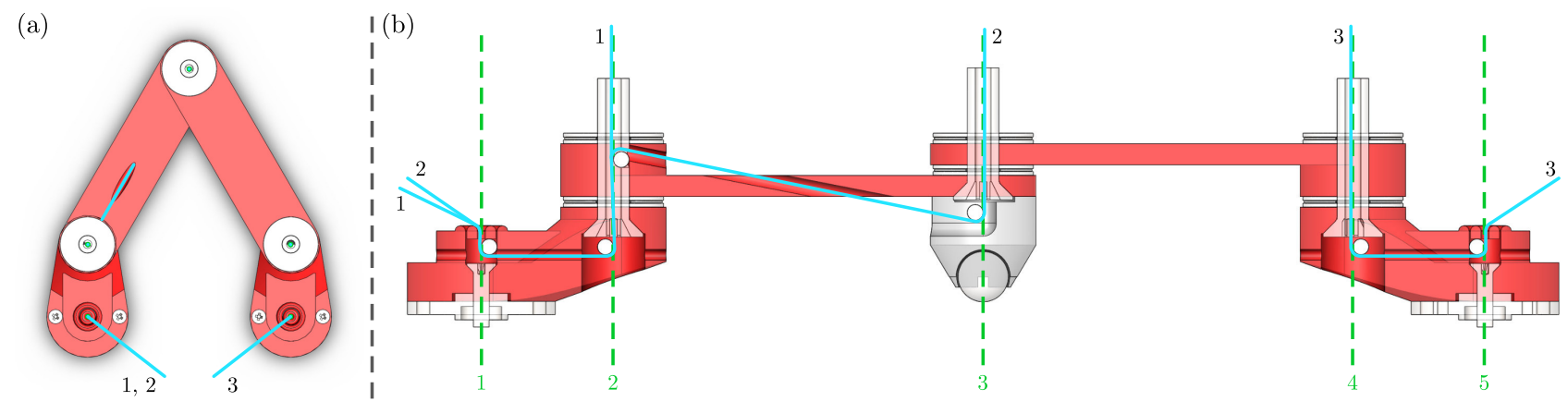

Fig. 2. CAD model showing the five-bar linkage design and configuration-independent tendon routing (blue lines 1,2 , and 3 ), achieved by aligning tendon routing with the 5 axes of rotation (green): (a) Top-view showing five-bar initial configuration and (b) Unwrapped section-view showing tendon routing.

transmission mechanism, have less actuators and are simpler to control while being able to grasp diverse objects [8, 9]. For example, the Velo gripper [10] can perform both parallel and fingertip grasps with a single actuator, being able to pick up small objects off a flat surface. The Ocean One hand [11] achieves a variety of pinch and power grasps via elastic finger joints and a spring transmission. Catalano et al. [12] proposed an adaptive synergy that allows the 19-joint hand to accommodate an arbitrary number of grasp postures using only one actuator. In general, underactuated hands, by incorporating elastic and passive elements to generate self adaptation for dealing with uncertainties, have been well developed for grasping tasks. However, these characteristics and hands have been seldom leveraged for achieving dexterous manipulation while keeping control complexity low.

Several robotic hands have been developed by modifying existing underactuated designs in different ways to achieve translation and rotation of objects. Chavan-Dafle et al. [13] designed a pneumatic shape-shifting fingertip to enable a simple parallel jaw gripper to reorient and grasp objects by changing the contact type between the fingertips and objects. This method takes advantage of gravity to reorient the object, which makes the direction and range of rotation limited. For increasing rotation capabilities, elastic pivot joints between the fingers can be implemented [14] or soft fingertips can be used as it has been shown that, when compare to rigid fingertips, they generate a larger manipulation workspace for a given gripper [15]. Indeed, by adding inflatable air cavities in soft fingertips [16], simple grippers can in-hand manipulate (soft and delicate) objects against gravity. Alternatively, by dynamically varying the surface material of fingers both translation and rotation can be achieved [17].

Adding an extra degree of freedom on the proximal joints of an underactuated robotic hand is also a popular method to increase hand dexterity, without increasing the actuator space excessively [18-20]. For instance, the iHY Hand [18] is a three-fingered underactuated hand driven by 5 actuators in which two of the fingers have a coupled adduction/abduction motion at the proximal joints to perform different grasps and simple re-position tasks. Another alternative is to change the morphology of the fingers to achieve a particular motion characteristic. Ma et al. [21] proposed a curved finger design to a three-fingered underactuated hand for objects to follow a sphere surface, regardless of the object size and grasping location. However, in all these cases, the control simplicity that underactuation gives to grasping is not inherited or maintained when performing in-hand manipulation operations.

Similar to our work is the approach to enlarge the grasping capabilities of robot hands based on changing the relevant position of the proximal joints of the hand, which is equivalent to equip it with a reconfigurable palm [22-25]. For instance, two of the articulated fingers of the BarrettHand [22] can rotate $180^{\circ}$ around the central axis of the palm to adapt various grasping configurations. This design approach is adopted in [24] and [25] to achieve some particular manipulation tasks. Regarding reconfigurable palms, Dai et al. proposed the Metahand [23] which uses a spherical mechanism as a palm, proposing later a design based on a planar linkage [26]. These works are the closest to our approach, which is also based on incorporating a reconfigurable palm in the robot hand system. However, while the hardware may seem similar, our ethos and objectives are completely different, as rather than interested in presenting the versatility of a new hand, our focus is on investigating how robot hand hardware characteristics, such as a reconfigurable palm, can be leveraged to devise simple algorithms for planning and control of in-hand manipulation operations with arbitrary objects.

In this paper, we introduce the Reconfigurable Underactuated constant-Tendon Hand (RUTH) gripper, a three-fingered self-adaptive reconfigurable underactuated hand which decouples grasping and manipulation to achieve systematic prehensile in-hand manipulations regardless of object size or shape. The hand utilises a two-degree-of-freedom five-bar linkage as the palm of the gripper, having a total of three actuatorstwo for controlling the reconfiguration of the palm and one for controlling the underactuated fingers, which are connected to the mobile revolute joints of the palm. The reconfigurable palm provides the hand with large grasping versatility, while allowing the easy computation of an object-invariant map between task space and joint space for manipulation. Using this map, the in-hand manipulation of objects of different sizes and shapes from one pose to another is straightforward and 
systematic, provided the objects are kept grasped. This step is guaranteed independently by the actuator that controls the underactuated fingers using a novel tendon routing that eliminates tendon length variations when the palm reconfigures.

In what follows, we begin by discussing the design and prototype of the RUTH gripper. We then analyse its grasping and manipulation characteristics, presenting algorithms for computing the mapping between object manipulation workspace and active joint space, and for computing the shortest distance in joint space to move a grasped object from its current pose to a desired one. Next we evaluate the gripper performance on both grasping and in-hand manipulation tasks. Lastly we discuss the comparison between the simulation and the gripper performance as well as future work.

\section{Design of the Reconfigurable Palm GripPer}

\section{A. Five-Bar Reconfigurable Palm}

A five-bar linkage was selected for reshaping the gripper palm as it allowed 3 specific points, namely five-bar joints 2,3 , and 4 , to be repositioned in the $x-y$ plane through the control of only two motors. When selecting dimensions for the five-bar linkage, a symmetrical structure (link $1=$ link 4 , link $2=$ link 3) was chosen to ease manufacturing and further to allow for the five-bar to form an equilateral triangle. This was achieved by also distancing the motors axes (link 5) the same distance as between the five-bar joint axes 2 and 3 (link $2=$ link $3=$ link 5). The isolated five-bar linkage in its equilateral triangle configuration can be seen in Fig2 (2). To ensure the five-bar was supported throughout its manipulation, a caster wheel was placed under joint 3 . As the caster wheel required a surface to translate on, the magnitude of the 5-bar was limited by the size of the gripper housing, which was limited by the size of the motors used. Through careful positioning of the 3 servo motors (Dynamixel MX64), a compact housing size of $140 \mathrm{~mm} \oslash$ (by $66 \mathrm{~mm}$ tall) was developed. From this size, links 2, 3, and the motor distance (link 5) were set as $70 \mathrm{~mm}$. To provide structural rigidity the links were given cross-sectional dimensions of $20 \mathrm{~mm} \times 4 \mathrm{~mm}$ (minimum). Shorter links 1 and 4 were dimensioned such that each motor could theoretically achieve full $360^{\circ}$ motion without collisions, and with a link width of $20 \mathrm{~mm}$ this gave a resulting length of $25 \mathrm{~mm}$. Between each of the contacting faces of the links, roller bearings $(20 \mathrm{~mm} \oslash)$ were used to reduce the friction of the system.

To actuate the fingers of the underactuated gripper, a tendonbased method was implemented as this allowed all 3 fingers to be controlled using a single motor. However, one of the unique features this gripper presents is the variation in distance between the fingers and actuation motor as the five-bar linkage changes configurations. In typical underactuated grippers, the tendon exits the base of each finger and connects directly to the actuation motor. If this were to be implemented with the RUTH gripper, as the five-bar changes its configuration the finger tendons would vary in length relative to each other, and grasping would fail. To overcome this issue, a constant-tendon system was implemented, where the length of each finger

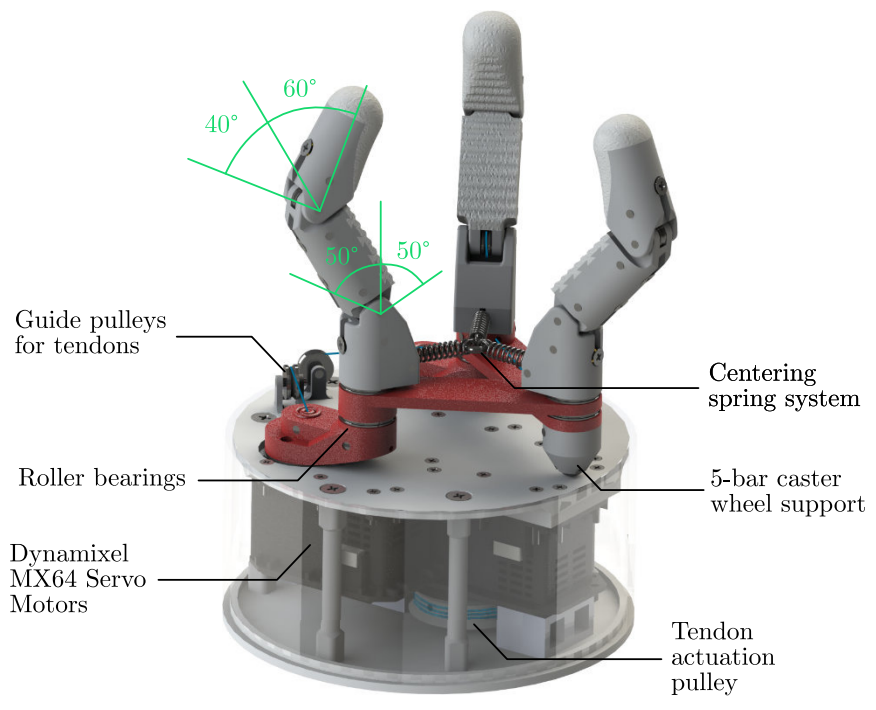

Fig. 3. CAD model showing the overall gripper structure and components. Finger joint angle limits are also shown.

tendon was independent of the five-bar configuration. Each of the 3 tendons were passed through the five-bar mechanism, ensuring no horizontal translation occurred across the fivebar joint axes. Instead, each tendon was constrained to only vertical translation across joint axes through the use of $3 \mathrm{~mm}$ steel pins as reduced-friction guide pulleys. A cross section of the expanded 5-bar linkage showing the tendon routing for the 3 individual tendons can be seen in Fig 2 (b). Once the tendons exited the five-bar linkage at joints 1 and 5, they were routed to the inverted actuation motor in the beneath housing using guide pulleys. These guide pulleys and other components of the gripper can be seen in Fig. 3

\section{B. Underactauted Fingers}

To affix the fingers to the five-bar linkage, while also allowing rotation of the fingers, a $6 \mathrm{~mm}$ machine screw was threaded through the joint axis of each finger. The machine screw was also hollowed out to allow the tendon to pass directly through the axes, with the aforementioned no horizontal translation. The three fingers followed an identical design, with 2 flanges providing $\pm 50^{\circ}$ motion for the proximal flange and $+60^{\circ}$ $40^{\circ}$ motion for distal flange, shown in Fig. 3 . To increase the grasping ability, the surfaces of the fingers were coated in textured silicone (SmoothOn Eco-Flex 00-10).

To maintain the grasping capability as the five-bar configuration changes, the fingers actuation motion should be towards the centre of the triangle formed by the three finger base positions (five-bar axes 2, 3, and 4). To achieve this, the direction of each finger was controlled by a high stiffness spring attached at the base of each finger to a central ring. The central ring is held in the triangle centre by the three finger springs, and the ring design allows each of the finger springs to rotate around the centre without experiencing the torsion 
(a)

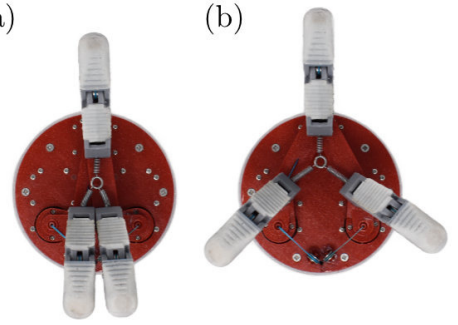

(c)

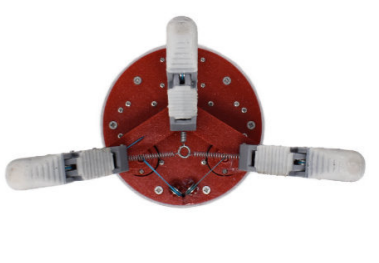

Fig. 4. Different types of grasp achievable with the RUTH gripper: (a) Parallel, (b) Trigonal planar, and (c) T-shape.

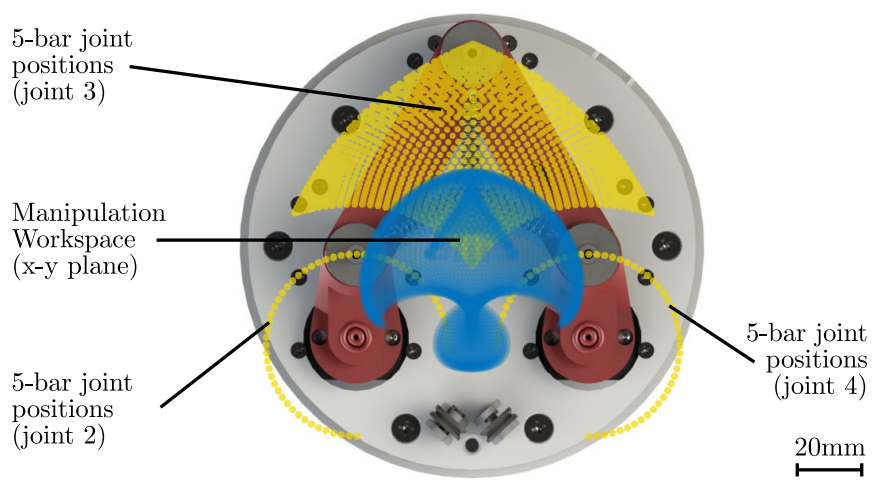

Fig. 5. X-Y manipulation workspace of the RUTH gripper (blue) in respect to the five-bar mechanism and gripper housing (fingers removed for clarity). Five-bar joint positions for joints 2, 3, and 4 are also shown (yellow).

expected with fixed springs. This spring system can be seen in Fig. 3 .

\section{Design of the Prototype}

The prototype was constructed mostly from 3D printed parts on a single nozzle desktop 3D printer. The fingers were printed out of Polylactic Acid (PLA), while the five-bar was printed out of Polyethylene Terephthalate Glycol (PETG) for increased rigidity. The housing for gripper was constructed from a combination of PLA and PETG printed parts. To ensure a uniform surface on the top of the housing for the caster wheel, all surface fixtures were filled with hot glue and smoothed till flat. An Arduino Nano microcontroller was used to control the hand, utilising a software serial connected tristate buffer (74LS241N) to communicate using half-duplex UART protocol with the Dynamixel MX64 servo motors. Thanks to the small size of the electronics, they were contained within the gripper housing.

\section{Grasping And Manipulation Analysis of the GRIPPER}

\section{A. Grasping Configuration}

We first explore the different grasping capabilities of the RUTH gripper. Using the five-bar structure, we can reposition the fingers to allow for a variety of grasps, shown in Fig. 4. In its default configuration, an equilateral triangle, the fingers form a trigonal planar grasp, ideal for power grasping spherical objects [Fig. 4(b)]. By rotating the motors inwards, the two short-link fingers come together to form a single 'finger', forming a parallel grasp with the long-link finger [Fig. 4(a)]. This grasp is ideal for pinch grasping small objects and planar grasping regular cubic objects. Finally, by rotating the motors outwards the five-bar expands and the gripper forms a T-shape grasp, where the short-link fingers are parallel and opposite each other, with the long-link finger acting perpendicular [Fig. 4(c)]. The enlarged reach of this grasp enables the grasping of larger objects, and is a combination of both the parallel and trigonal planar grasp in that it can perform power grasps on the majority of objects, with an increase in performance grasping regular cubic objects over the trigonal planar due to the $90^{\circ}$ rotated fingers, rather than $120^{\circ}$.

\section{B. Feasible Grasping Workspace}

The feasible grasping workspace of the gripper is the set of positions in which an object can lie relative to the base of the hand, the palm, and be successfully grasped. It is possible to achieve a range of different grasping positions as the five-bar linkage can be reconfigured such that the centre point of the proximal joints of the fingers is moved underneath the position of the centre of the object. The two-dimensional grasping workspace is therefore given by the set of positions that the centre point of the proximal joints of the fingers, namely $\mathbf{P}_{2}$, $\mathbf{P}_{3}$, and $\mathbf{P}_{4}$, can get.

The positions of the palm's base joints, namely $\mathbf{P}_{1}$ and $\mathbf{P}_{5}$, are known and the positions of joints $\mathbf{P}_{2}$ and $\mathbf{P}_{4}$ are determined by the input angles of the actuators, say $\theta_{1}$ and $\theta_{2}$, such that

$$
\begin{aligned}
& \mathbf{P}_{2}=\mathbf{P}_{1}+l_{1}\left[\cos \theta_{1} \sin \theta_{1}\right]^{T} \text { and } \\
& \mathbf{P}_{4}=\mathbf{P}_{5}+l_{1}\left[\cos \theta_{2} \sin \theta_{2}\right]^{T} .
\end{aligned}
$$

The position of $\mathbf{P}_{3}$ can be then obtained using bilateration [27 28] as

$$
\mathbf{P}_{3}=\mathbf{P}_{2}+\mathbf{Z}_{2,4,3}\left(\mathbf{P}_{4}-\mathbf{P}_{2}\right)
$$

where

$$
\mathbf{Z}_{2,4,3}=\frac{1}{2 d_{2,4}^{2}}\left[\begin{array}{cc}
d_{2,4}^{2} & -4 A_{2,4,3} \\
4 A_{2,4,3} & d_{2,4}^{2}
\end{array}\right],
$$

with $A_{2,4,3}=\frac{1}{4} \sqrt{\left(d_{2,4}^{2}+2 l_{2}^{2}\right)^{2}-2\left(d_{2,4}^{4}+2 l_{2}^{4}\right)}$ and $d_{i, j}$ being the distance between $\mathbf{P}_{i}$ and $\mathbf{P}_{j}$. The sign of $A_{2,4,3}$ determines whether $\mathbf{P}_{3}$ lies to the left or the right of the vector from $\mathbf{P}_{2}$ to $\mathbf{P}_{4}$; herein, the sign of $A_{2,4,3}$ is positive as it is desired that $\mathbf{P}_{3}$ lies always to the left.

The centre point, $\mathbf{C}$, of the proximal joints of the fingers is then given by

$$
\mathbf{C}=\frac{\mathbf{P}_{2}+\mathbf{P}_{3}+\mathbf{P}_{4}}{3} \text {. }
$$

The numerical grasping workspace of the gripper can be obtained by sweeping through the possible input actuator angles, $\theta_{1}$ and $\theta_{2}$, and computing the set of positions of $\mathbf{C}$ using equations (1)-(4). The only mechanical constraint that needs to be taken into account is the links cannot collide with 

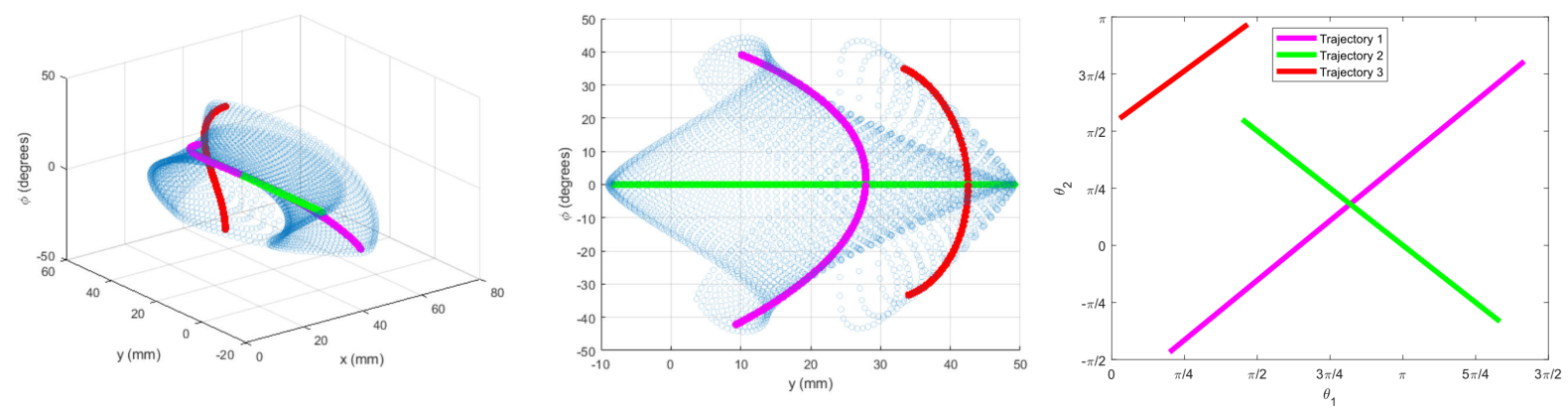

Fig. 6. The manipulation map with three example trajectories of the grasped object. (a) and (b) show two different views of the object trajectories and (c) shows the corresponding joint angle profiles.

the tendons passing into the base joints $\mathbf{P}_{1}$ and $\mathbf{P}_{5}$. Following this, if $\theta_{1}$ and $\theta_{2}$ are defined as the angles taken anti-clockwise from the $x$-axis to the vectors from $\mathbf{P}_{1}$ to $\mathbf{P}_{2}$, and $\mathbf{P}_{5}$ to $\mathbf{P}_{4}$, respectively, then the limits to avoid collisions with the tendons are $0<\theta_{1}<3 \pi / 2$ and $-\pi / 2<\theta_{2}<\pi$. The computed workspace is shown in Fig. 5, where an instance of the fivebar linkage is also given for perspective.

\section{Systematic In-hand Manipulation Map and Planning}

In this section, the prehensile in-hand manipulation capabilities of the gripper are demonstrated. Firstly, a manipulation map is generated which relates the planar position of the centre of the object and its orientation to the configuration of the fivebar linkage. Algorithm 11 describes the method of computing the mapping between the object manipulation workspace and the active joint space, which describes the possible combinations of $\theta_{1}$ and $\theta_{2}$. All the feasible combinations of $\theta_{1}$ and $\theta_{2}$ are then swept through and the centre of the object, whose coordinates are given by $x$ and $y$, is determined using the method described in section III-B.

Additionally, the orientation of the object, denoted by $\phi$, is given by the anti-clockwise angle from the $x$-axis to the vector from $\mathbf{P}_{2}$ to $\mathbf{P}_{4}$; this is computed using the two-argument inverse tangent function so that the direction of the angle is determined. Each feasible object pose is stored in the $k^{\text {th }}$ row of matrix $\mathbf{M}_{1}$ and the corresponding joint angles make up the $k^{\text {th }}$ row of $\mathbf{M}_{2}$. As the units of $\mathbf{M}_{1}$ are not homogeneous, $\mathbf{M}_{1}$ is normalised, such that

$$
\mathbf{M}_{1, \text { norm }, i}=\left[\begin{array}{c}
\frac{x_{i}-\min (x, y)}{\max (x, y)-\min (x, y)} \\
\frac{y_{i}-\min (x, y)}{\max (x, y)-\min (x, y)} \\
\frac{\phi_{i}-\min (\phi)}{\max (\phi)-\min (\phi)}
\end{array}\right]^{T}
$$

where $\mathbf{M}_{1, \text { norm, } i}$ denotes the $i^{\text {th }}$ row of the normalised $\mathbf{M}_{1}$ matrix, $\left(x_{i}, y_{i}, \phi_{i}\right)$ make up the $i^{t h}$ row of $\mathbf{M}_{1}$, $\min (x, y) / \max (x, y)$ denotes the minimum/maximum of all $x$ and $y$ values, and $\min (\phi) / \max (\phi)$ denotes the minimum/maximum of all $\phi$ values.

Algorithm 2, the computation of manipulation planning, utilises the above mapping to find the shortest distance in joint space to move from the current pose of the manipulator to the desired pose, $\mathbf{D}=\left(x_{D}, y_{d}, \phi_{D}\right)$. $\mathbf{D}$ is normalised in the same manner as $\mathbf{M}_{1, n o r m}$, such that

$$
\mathbf{D}_{\text {norm }}=\left[\begin{array}{c}
\frac{x_{D}-\min (x, y)}{\max (x, y)-\min (x, y)} \\
\frac{y D-\min (x, y)}{\max (x, y)-\min (x, y)} \\
\frac{\phi D-\min (\phi)}{\max (\phi)-\min (\phi)}
\end{array}\right]^{T} .
$$

Now, in order to find the nearest neighbour in $\mathbf{M}_{1, \text { norm }}$ to $\mathbf{D}_{\text {norm }}$, a $k$ - $d$ tree is formed from $\mathbf{M}_{1, \text { norm }}$. This tree is formed by taking the median of the points in $\mathbf{M}_{1, \text { norm }}$ with respect to a particular coordinate (this point is called the root), and splitting the set into two; the subset of points to the left of the root comprise the left side of the tree and the ones to the right comprise the right side of the tree. The median of each of these sets is found with respect to the next coordinate and the tree is formed by continuing to partition all of the points in this fashion. The $k-d$ tree is then used to perform a nearest neighbour search, such that the point in $\mathbf{M}_{1, \text { norm }}$ that is the shortest Euclidean distance from $\mathbf{D}_{\text {norm }}$ is found. This is performed by starting at the root and moving down the tree depending on whether the coordinate of the desired point corresponding to the current partition is to the left or the right of the partition.

If a point in the tree is reached which is closest so far to the desired point, it is recorded as such. The possibility that there are points on the other side of the partition that are closer is checked by forming a sphere around the desired point with a radius equal to that of the distance between the current closest point and the desired point-if the sphere crosses the partitioning plane, there could be closer points and therefore the opposite branch must be checked, otherwise the opposite branch can be neglected. This algorithm continues until the nearest neighbour is found. The index, $m$, of this point is taken and the final joint coordinates, $\left(\theta_{F, 1}, \theta_{F, 2}\right)$, are given by the $m^{\text {th }}$ row of $\mathbf{M}_{2}$, the matrix of joint angles.

The path from the current pose of the manipulator, defined by the joint angles $\left(\theta_{C, 1}, \theta_{C, 2}\right)$, to the final pose is discretised such that the $\theta_{1}$ and $\theta_{2}$ step values are constant and equal in number. Fig. 6 shows the manipulation map of the gripper with 3 examples trajectories of the object; Fig. 6(a) and Fig. 6(b) show the trajectories of the object from two different views and Fig. 6(c) shows the corresponding joint angle profiles. 

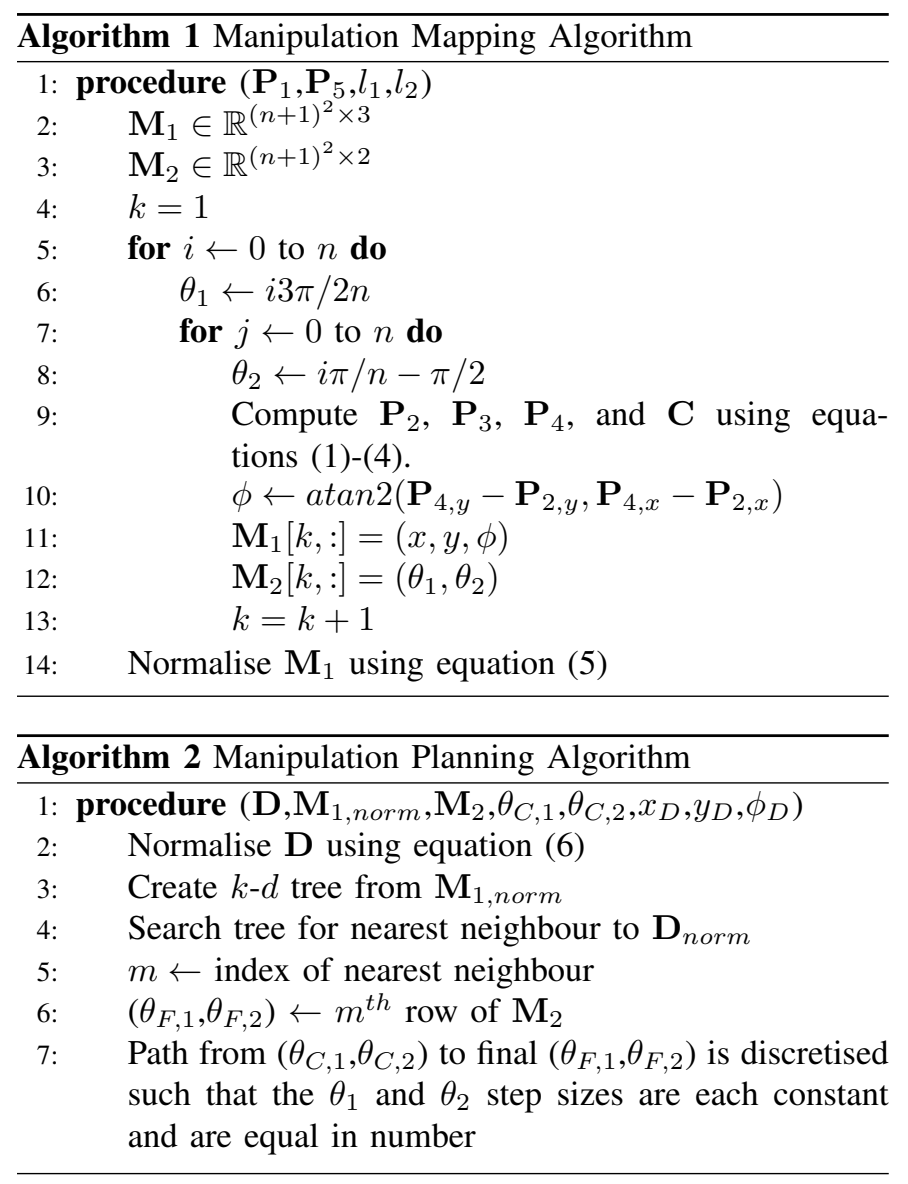

\section{PERformance Evaluation}

To show how the five-bar linkage of the RUTH Gripper impact the grasping and in-hand manipulation behaviour, a series of YCB objects [29] was used for grasping and systematic inhand manipulation tasks. These tasks included pick up and grasping from workbench and in the air with three grasping configurations. Nine objects of various sizes and shapes were used for systematic in-hand translation and rotation tasks. This section highlights the five-bar reconfigurable palm and other design features illustrated by the experiments. A video from the experiments is included with this paper in the supplementary material.

\section{A. Experimental Setup}

The RUTH Gripper was attached to a Universal Robot Arm (UR5) for performing grasping tests. Arduino Nano was used to control the movement of the five-bar linkage (reconfigurable palm) and the grasping through the Dynamixel motors. The size of the six testing cylindrical objects vary from $30 \mathrm{~mm}$ to $90 \mathrm{~mm}$, the three additional testing objects correspond to a cube, a hexagonal prism, and a triangular prism all of size 50mm (see Fig. 7). Motion tracking cameras (OptiTrack Flex3) were used to track the testing object trajectories. All these objects had four tracking markers on them. Each in-hand manipulation test consisted of 5 repeated trials to generate reliable performance results.

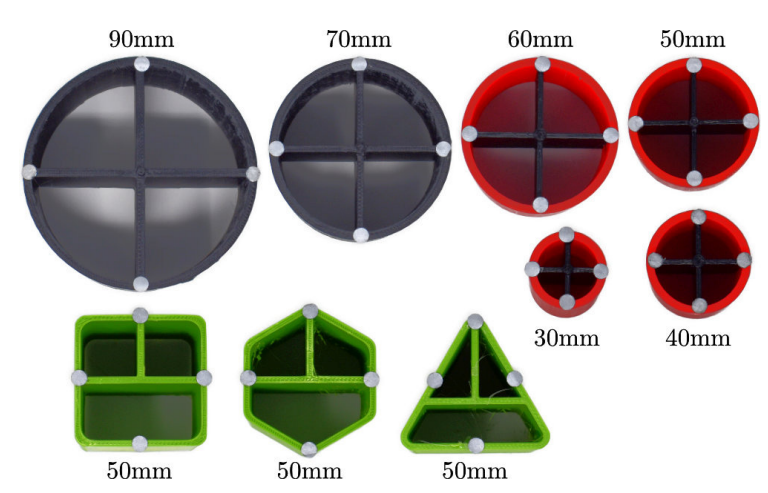

Fig. 7. Objects used to evaluate the in-hand manipulation of the gripper.

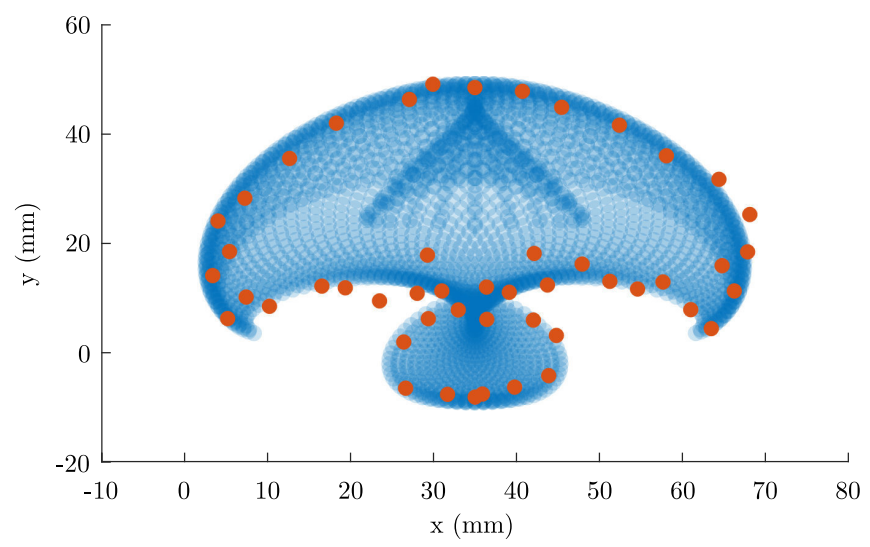

Fig. 8. Experimental object positions at the RUTH gripper's configuration boundary (red). Simulation workspace of the RUTH gripper (blue).

\section{B. Grasping Capability and Workspace}

By taking advantage of the five-bar linkage palm, the RUTH gripper is able to grasp various objects in the different grasping configurations shown in Fig. 4 The grasping capability of the RUTH gripper was tested by grasping a range set of YCB objects which include a set of spheres ranging in size from $17.4 \mathrm{~mm}$ to $145 \mathrm{~mm}$, a set of kitchen objects, and fruits. The gripper showed strong adaptability of grasping various sizes and shapes of objects. Experiments showed that for flat objects, the parallel grasp performed better; for large objects (greater than the palm size $(70 \mathrm{~mm})$ ), the T-shape grasp performed better.

The grasping workspace was also measured by grasping the $50 \mathrm{~mm}$ cylindrical object at the boundary case and compared to the simulated workspace shown in Fig. 8. The blue dots are the feasible manipulation workspace of the RUTH gripper in $\mathrm{X}-\mathrm{Y}$ plane produced by MATLAB. The red dots are the motion tracking data of the grasped object centre positions. The experimental data verified the gripper grasping workspace with a little deviation due to the underactuated finger design and inconsistent grasping force. 
(a)

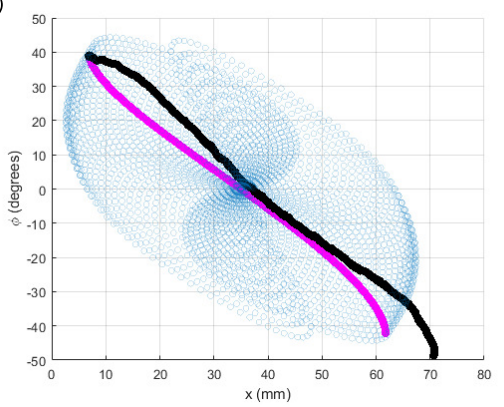

(b)

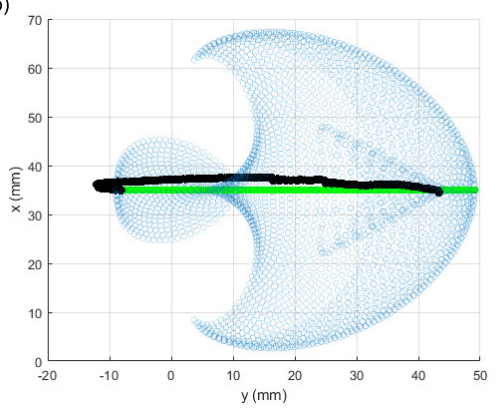

(c)

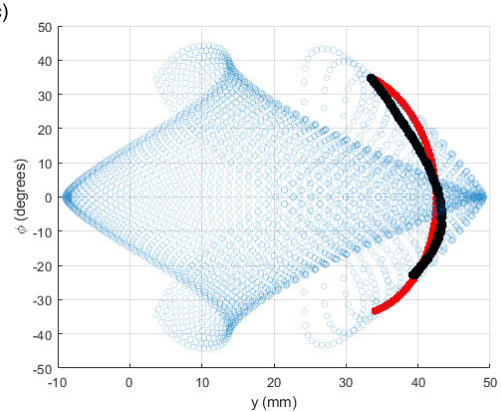

Fig. 9. Experimental motion tracking object trajectories (black) overlaid on simulated trajectories.

TABLE I

SIMULATION AND EXPERIMENTAL TRANSLATION AND ROTATION AVERAGE RESULTS OF 3 TRAJECTORIES ON VARIOUS SIZE AND SHAPE OBJECTS

\begin{tabular}{c|cc|cc}
\hline \hline & \multicolumn{2}{|c|}{ Simulation } & \multicolumn{2}{c}{ Experimental (error) } \\
\hline & Translation $(\mathrm{mm})$ & Rotation & Average $T$ & Average $R$ \\
\hline T1 & 54.9 & $81.2^{\circ}$ & $55.1(0.3 \%)$ & $72.2^{\circ}(5 \%)$ \\
\hline T2 & 57.2 & $0^{\circ}$ & $50.2(10 \%)$ & $4^{\circ}(2 \%)$ \\
\hline T3 & 0.7 & $68.2^{\circ}$ & $14.4(19 \%)$ & $55.8^{\circ}(7 \%)$ \\
\hline \hline
\end{tabular}

\section{Systematic Prehensile In-hand Manipulation}

As proposed in section III.C, the prehensile in-hand manipulation map of the gripper was generated in terms of $x, y$ and $\phi$ using Algorithm 1. The grasped object can be moved into a desired pose by using the map to identify the $\theta_{1}$ and $\theta_{2}$ values as described in Algorithm 2. Three characteristic trajectories of the object were chosen for the tests. During the tests, the gripper were given the $\theta_{1}$ and $\theta_{2}$ values produced by the map by input the target $x, y$ and $\phi$. The change of the $\theta_{1}$ and $\theta_{2}$ during the manipulation are linear as shown in Fig. 6(c). Fig. 9 showed the sample experimental object trajectories compared to the simulation trajectories. It showed not only the end position, but the whole trajectory during manipulation. Table I] shows the numerical and experimental translation and rotation values for those three chosen trajectories with percentage errors. The experimental translation and rotation results are the average results of all the 9 objects over the 5 trials.

For the first trajectory, a random end position was chosen, the average experimental results were close to the desired position with translation of $55.1 \mathrm{~mm}$ compared to $54.9 \mathrm{~mm}$ and the rotation of $72.2^{\circ}$ compared to $81.2^{\circ}$. The errors are less than $5 \%$. The second trajectory was chose to perform a pure translation trajectory with no rotation. The gripper had average $50.2 \mathrm{~mm}$ in translation compared to the desired 57.2 $\mathrm{mm}$ with error around $10 \%$, and had a $4^{\circ}$ deviation on rotation. For the third trajectory, approximate pure rotation trajectory was selected with $0.7 \mathrm{~mm}$ for translation. The error range of both translation and rotation of this trajectory increased with translation from $0.7 \mathrm{~mm}$ to $14.4 \mathrm{~mm}$ and rotation from $68.2^{\circ}$ to $55.8^{\circ}$. The gripper showed worse accuracy at extreme conditions (boundary positions and decoupled motions).

Additionally, the translation and rotation errors for each

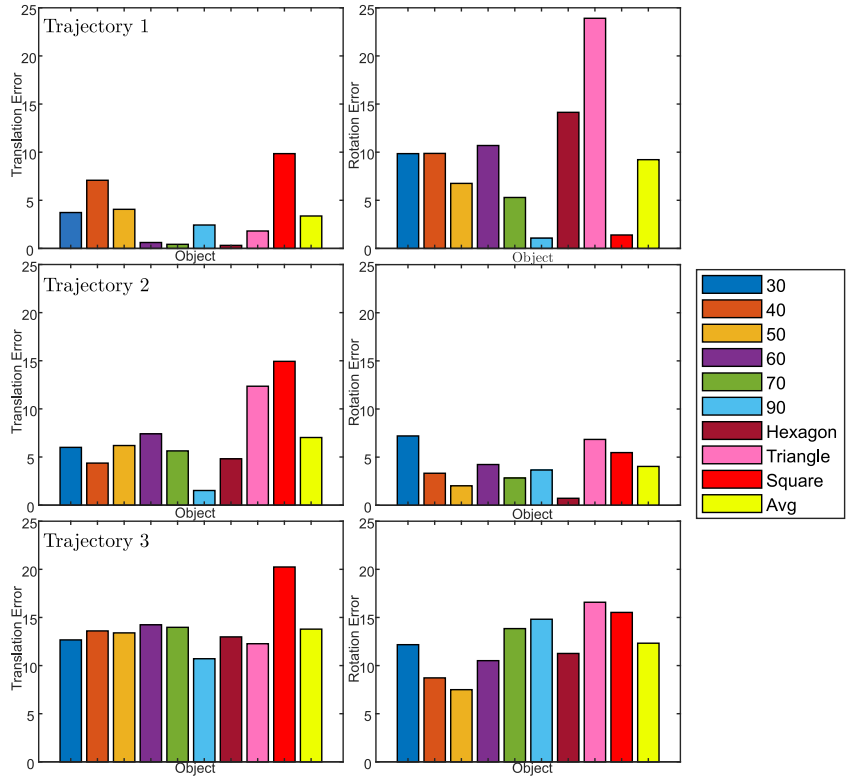

Fig. 10. Translation and rotation errors of the experimental results for each trajectory on 9 objects. Different colours indicate different objects. The last bright yellow indicate the average error of all 9 objects on each trajectory.

testing object were plotted in Fig. 10. Most of the errors are less than $15 \mathrm{~mm}$ and $15^{\circ}$ for translation and rotation, respectively, apart from the rotation error for the triangular prism. It showed that the errors for the third trajectory are higher than for the other two. However, the error differences of the third trajectory are similar for different objects. It might indicate that this gripper design may have imperfections for certain manipulation trajectories that reach boundary conditions. Throughout all three trajectories, the average error differences between different objects are less than $8.5 \%$.

\section{DISCUSSION}

The overall performance of the RUTH gripper on manipulating an object is predictable with some deviations, especially for non-cylindrical objects. For non-cylindrical objects, the distances from each finger contact point to the centre of the object are different during the manipulation, which is a challenge for an underactuated gripper. Those manipulation errors may occur because of the gripper grasping force is 
not strong enough to push the manipulated object towards the centre of the gripper. This may easily affect the final position of the object and produce errors in translation range. For the proposed design, in order to have a constant tendon routing design for the five-bar linkage (the reconfigurable palm) the tendon routing shown in Fig. 2 may reduce the force transmission efficiency significantly given the small-radii pulleys used. The motor we used is limited on the output torque at around $5.8 \mathrm{Nm}$ which is near the torque that required to actuate the fingers from the starting position. A stronger motor may improve the accuracy of the gripper.

The final position errors may occur because of the changing contact points during the prehensile in-hand manipulation. In the simulation, we assumed that the object is always being grasped at the centre of the hand and the grasping configuration of each finger are identical. However, in reality, there are some aspects that make these assumptions not totally valid. Firstly, in our gripper design, the three threephalanx fingers are actuated by only one motor via tendons. Although, we optimised the tendon routing method for the fingers to perform proximal joints bending first and then the distal joints. With the same amount of tendon length change, each finger configuration may still vary due to the contact force, manufacture errors, and structure friction, to name some. With this uncertainty, those three fingers may end up with slightly different bending height which will cause object rotation in both $x$ and $y$ directions. Secondly, the contact points between the fingertips and the objects are changing all the time during the manipulation. During these changes, rolling occurs easily and may affect the performance of the objects. So far, considering those parameters into the simulation is still an open research question.

To maintain the grasping capability as the five-bar configuration changes, the fingers actuation motion should be towards the centre of the triangle formed by the three finger base positions proposed in section II. To achieve this, the direction of each finger was controlled by a high stiffness spring attached at the base of each finger to a central ring. In some cases, when the gripper grasped an object tightly, the central ring may struggle to pull all the fingers towards the centre of the triangle which may produce the position error adding to the end position.

As mentioned in the performance evaluation, the gripper performance on manipulating various objects of different sizes and shapes is similar. Fig. 10 shows that the cylindrical object of size $90 \mathrm{~mm}$ has the least average error for all three trajectories. This may be the case because the errors produced by the contact points changing or rolling can be relatively small compare to the objects of larger size. For instance, with the same amount of errors, small objects will have a greater influence than the larger objects. Additionally, fingers are bending less to grasp larger objects, which may decrease the probability of uncertainties. Moreover, the contact conditions are not stable for triangular prisms and cubes, especially grasping at the corners. Overall, the object size is not the dominant parameter for the accuracy of the performance. The object shape may vary the accuracy at certain conditions. However, results demonstrate that by generating the manipulation map, the gripper can perform systematic prehensile in-hand manipulation for different sizes and shapes. It is also important to highlight that with the proposed mechanical-intelligence design principle, this in-hand manipulation is achieved with a simple control algorithm and a minimum number of actuators.

\section{CONCLUSION}

In this paper we presented the design, construction, and evaluation of a reconfigurable underactuated constant-tendon hand (the RUTH gripper), which decouples manipulation and grasping to facilitate the control and implementation of prehensile in-hand manipulation. Through the use of a fivebar linkage as the palm of the gripper, the fingers are capable of repositioning to allow not just different grasp types, but object-invariant in-hand manipulation. We explored the design of the reconfigurable palm and how we achieved underactuated constant-tendon routing despite the ability of the fingers to change its proximal joint position. We proposed an algorithm to compute the feasible manipulation map using distancebased kinematics, and also proposed an algorithm for manipulation planning and control. We experimentally evaluated the hand in both grasping and in-hand manipulation capabilities. A wide range of objects were tested for the grasping capability. Nine objects of different size and shape were manipulated in three trajectories using the algorithms proposed. From the results we see that with the proposed mechanical-intelligence design principle, the gripper can achieve precise systematic inhand manipulation regardless the particularities of the object with a simple control scheme. For future work, force analysis can be performed to calculate the required torque for the constant-tendon routing, and this routing can be optimised to improve the force transmission efficiency. Moreover, the introduced grasping-manipulation decoupling approach can be explored for 6D in-hand manipulation.

\section{ACKNOWLEDGEMENT}

This work was supported in part by the Engineering and Physical Sciences Research Council grant EP/R020833/1 and an Amazon Research Award.

\section{REFERENCES}

[1] J. K. Salisbury and J. J. Craig, "Articulated hands: Force control and kinematic issues," The International Journal of Robotics Research, vol. 1, no. 1, pp. 4-17, 1982.

[2] S. Jacobsen, E. Iversen, D. Knutti, R. Johnson, and K. Biggers, 'Design of the Utah/MIT dextrous hand," in International Conference on Robotics and Automation, vol. 3. IEEE, 1986, pp. 1520-1532.

[3] R. Walkler, "Developments in dextrous hands for advanced robotic applications," in World Automation Congress, 2004, pp. 123-128.

[4] A. Schmitz, U. Pattacini, F. Nori, L. Natale, G. Metta, and G. Sandini, 'Design, realization and sensorization of the dexterous iCub hand," in International Conference on Humanoid Robots. IEEE, 2010, pp. 186-191. 
[5] I. Akkaya, M. Andrychowicz, M. Chociej, M. Litwin, B. McGrew, A. Petron, A. Paino, M. Plappert, G. Powell, R. Ribas et al., 'Solving Rubik's Cube with a Robot Hand," arXiv preprint arXiv:1910.07113, 2019.

[6] O. M. Andrychowicz, B. Baker, M. Chociej, R. Jozefowicz, B. McGrew, J. Pachocki, A. Petron, M. Plappert, G. Powell, A. Ray et al., "Learning dexterous in-hand manipulation," The International Journal of Robotics Research, vol. 39, no. 1, pp. 3-20, 2020.

[7] A. Billard and D. Kragic, "Trends and challenges in robot manipulation," Science, vol. 364, no. 6446, p. eaat8414, 2019.

[8] A. M. Dollar and R. D. Howe, "Joint coupling design of underactuated hands for unstructured environments," The International Journal of Robotics Research, vol. 30, no. 9, pp. 1157-1169, 2011.

[9] G. Bai and N. Rojas, "Self-Adaptive Monolithic Anthropomorphic Finger with Teeth-Guided Compliant CrossFour-Bar Joints for Underactuated Hands," in International Conference on Humanoid Robots. IEEE, 2018, pp. 145-152.

[10] M. Ciocarlie, F. M. Hicks, R. Holmberg, J. Hawke, M. Schlicht, J. Gee, S. Stanford, and R. Bahadur, "The Velo gripper: A versatile single-actuator design for enveloping, parallel and fingertip grasps," The International Journal of Robotics Research, vol. 33, no. 5, pp. 753767, 2014.

[11] H. Stuart, S. Wang, O. Khatib, and M. R. Cutkosky, 'The ocean one hands: An adaptive design for robust marine manipulation," The International Journal of Robotics Research, vol. 36, no. 2, pp. 150-166, 2017.

[12] M. G. Catalano, G. Grioli, E. Farnioli, A. Serio, C. Piazza, and A. Bicchi, "Adaptive synergies for the design and control of the Pisa/IIT SoftHand," The International Journal of Robotics Research, vol. 33, no. 5, pp. 768782, 2014.

[13] N. Chavan-Dafie, K. Lee, and A. Rodriguez, 'Pneumatic shape-shifting fingers to reorient and grasp," in International Conference on Automation Science and Engineering. IEEE, 2018, pp. 988-993.

[14] N. Rojas, R. R. Ma, and A. M. Dollar, "The GR2 gripper: an underactuated hand for open-loop in-hand planar manipulation|" Transactions on Robotics, vol. 32, no. 3, pp. 763-770, 2016.

[15] Q. Lu and N. Rojas, "On Soft Fingertips for In-Hand Manipulation: Modeling and Implications for Robot Hand Design," Robotics and Automation Letters, vol. 4, no. 3, pp. 2471-2478, 2019.

[16] Q. Lu, H. Liang, N. Thrishantha, and N. Rojas, "Precise in-hand manipulation of delicate objects using soft fingertips with adaptive sensing and active deformation," Accepted by International Conference on Soft Robotics, 2020.
[17] Q. Lu, A. B. Clark, M. Shen, and N. Rojas, "An origami-inspired variable friction surface for increasing the dexterity of robotic grippers," IEEE Robotics and Automation Letters, vol. 5, no. 2, pp. 2538-2545, 2020.

[18] L. U. Odhner, L. P. Jentoft, M. R. Claffee, N. Corson, Y. Tenzer, R. R. Ma, M. Buehler, R. Kohout, R. D. Howe, and A. M. Dollar, 'A compliant, underactuated hand for robust manipulation," The International Journal of Robotics Research, vol. 33, no. 5, pp. 736-752, 2014.

[19] Robotiq, '3-Finger Adaptive Robot Gripper," 2016. [Online]. Available: https: //robotiq.com/products/3-finger-adaptive-robot-gripper? 301=/en/products/industrial-robot-hand

[20] Righthand Robotics, 'ReFlex Robotic Gripper," 2019. [Online]. Available: https://www.labs.righthandrobotics. com/reflexhand

[21] R. R. Ma, N. Rojas, and A. M. Dollar, "Spherical hands: Toward underactuated, in-hand manipulation invariant to object size and grasp location," Journal of Mechanisms and Robotics, vol. 8, no. 6, p. 061021, 2016.

[22] W. Townsend, 'The BarrettHand grasper-programmably flexible part handling and assembly|" Industrial Robot: An International Journal, vol. 27, no. 3, pp. 181-188, 2000.

[23] J. S. Dai, D. Wang, and L. Cui, "Orientation and workspace analysis of the multifingered metamorphic handMetahand," Transactions on Robotics, vol. 25, no. 4, pp. 942-947, 2009.

[24] J. Spiliotopoulos, G. Michalos, and S. Makris, 'A reconfigurable gripper for dexterous manipulation in flexible assembly," Inventions, vol. 3, no. 1, p. 4, 2018.

[25] M. Higashimori, H. Jeong, I. Ishii, M. Kaneko, A. Namiki, and M. Ishikawa, 'A new four-fingered robot hand with dual turning mechanism," in International Conference on Robotics and Automation. IEEE, 2005, pp. 2679-2684.

[26] X. Cui, J. Sun, X. S. Zhang, S. J. Xu, and J. S. Dai, "A Metamorphic Hand with Coplanar Reconfiguration," in International Conference on Reconfigurable Mechanisms and Robots. IEEE, 2018, pp. 1-7.

[27] N. Baron, A. Philippides, and N. Rojas, "A Novel Kinematically Redundant Planar Parallel Robot Manipulator With Full Rotatability," Journal of Mechanisms and Robotics, vol. 11, no. 1, 2019.

[28] N. Rojas, "Distance-Based Formulations for the Position Analysis of Kinematic Chains," Ph.D. dissertation, Universitat Politècnica de Catalunya, Barcelona, Apr. 2012. [Online]. Available: http://upcommons.upc.edu/ handle/2117/94613

[29] B. Calli, A. Singh, A. Walsman, S. Srinivasa, P. Abbeel, and A. M. Dollar, "The YCB object and model set: Towards common benchmarks for manipulation research", in International Conference on Advanced Robotics. IEEE, 2015, pp. 510-517. 